\title{
The German Aortic Valve Registry (GARY): A Nationwide Registry for Patients Undergoing Invasive Therapy for Severe Aortic Valve Stenosis
}
A. Beckmann ${ }^{1}$
C. Hamm ${ }^{2}$
H. R. Figulla ${ }^{3}$ J. Cremer ${ }^{4}$
K. H. Kuck ${ }^{5}$
R. Lange 6
R. Zahn ${ }^{7}$
S. Sack ${ }^{8}$
G. C. Schuler ${ }^{9}$
T. Walther ${ }^{10}$
F. Beyersdorf ${ }^{11}$
M. Böhm ${ }^{12}$
G. Heusch ${ }^{13}$
A. K. Funkat
T. Meinertz ${ }^{15}$
T. Neumann ${ }^{16}$
K. Papoutsis ${ }^{17}$
S. Schneider ${ }^{18}$
A. Welz ${ }^{19}$
F. W. Mohr ${ }^{14}$ for the GARY Executive Board

${ }^{1}$ Deutsche Gesellschaft für Thorax-, Herz- und Gefäßchirurgie [DGTHG], Berlin, Germany

2 Abteilung für Kardiologie, Kerckhoff-Klinik, Bad Nauheim, Germany

${ }^{3}$ Klinik für Innere Medizin I, Universitätsklinikum Jena, Germany

${ }^{4}$ Klinik für Herz- und Gefäßchirurgie, University Hospital SchleswigHolstein, Campus Kiel, Germany

${ }^{5}$ Kardiologie, Asklepios Klinik St. Georg, Hamburg, Germany

${ }^{6}$ Klinik für Herz- und Gefäßchirurgie, Deutsches Herzzentrum München, Germany

${ }^{7}$ Medizinische Klinik B (Kardiologie, Pulmologie und Angiologie), Klinikum der Stadt Ludwigshafen, Germany

${ }^{8}$ Klinik für Kardio-, Pneumologie u. Intensivmedizin, Städt.Klinikum München $\mathrm{GmbH}$, Klinikum Schwabing, Germany

${ }^{9}$ Klinik für Innere Medizin/Kardiologie, Herzzentrum Leipzig GmbH, Germany

${ }^{10}$ Abt. für Herz- und Thoraxchirurgie, Kerckhoff-Klinik Bad-Nauheim, Germany

${ }^{11}$ Abt. Herz- und Gefäßchirurgie, Universitäts-Herzzentrum FreiburgBad Krozingen, Germany

12 Innere Medizin III-, Kardiologie-, Angiologie, Universitätsklinikum des Saarlandes, Homburg, Germany

13 Institut für Pathophysiologie, Universitätsklinikum Essen, Germany

${ }^{14}$ Klinik für Herzchirurgie, Herzzentrum Leipzig GmbH, Germany

${ }^{15}$ Deutsche Herzstiftung e.V., Frankfurt am Main, Germany

16 Klinik für Kardiologie, Universitätsklinikum Essen, Germany

17 Deutsche Gesellschaft für Kardiologie, Herz- und Kreislaufforschung Düsseldorf, Germany

18 Institut für Herzinfarktforschung, Ludwigshafen, Germany

${ }^{19}$ Klinik und Poliklinik für Herzchirurgie, Rheinische F.-W.-Universität Bonn, Germany

Thorac Cardiovasc Surg 2012;60:319-325.
Address for correspondence and reprint requests Dr. Andreas Beckmann, Deutsche Gesellschaft für Thorax-, Herz- und Gefäßchirurgie [DGTHG], Langenbeck-Virchow-Haus Luisenstr. 58-59, Berlin 10117, Germany (e-mail: gf@dgthg.de). received

July 3, 2012

accepted after revision

July 11,2012
Copyright ( $\odot 2012$ by Thieme Medical Publishers, Inc., 333 Seventh Avenue, New York, NY 10001, USA. Tel: +1(212) 584-4662.
DOI http://dx.doi.org/ $10.1055 / \mathrm{s}-0032-1323155$. ISSN 0171-6425. 


\begin{abstract}
Background The increasing prevalence of severe aortic valve defects correlates with the increase of life expectancy. For decades, surgical aortic valve replacement (AVR), under the use of extracorporeal circulation, has been the gold standard for treatment of severe aortic valve diseases. In Germany $\sim 12,000$ patients receive isolated aortic valve surgery per year. For some time, percutaneous balloon valvuloplasty has been used as a palliative therapeutic option for very few patients. Currently, alternatives for the established surgical procedures such as transcatheter aortic valve implantation (TAVI) have become available, but there are only limited data from randomized studies or low-volume registries concerning long-time outcome. In Germany, the implementation of this new technology into hospital care increased rapidly in the past few years. Therefore, the German Aortic Valve Registry (GARY) was founded in July 2010 including all available therapeutic options and providing data from a large quantity of patients. Methods The GARY is assembled as a complete survey for all invasive therapies in patients with relevant aortic valve diseases. It evaluates the new therapeutic options and compares them to surgical AVR. The model for data acquisition is based on three data sources: source I, the mandatory German database for external performance measurement; source II, a specific registry dataset; and source III, a follow-up data sheet (generated by phone interview). Various procedures will be compared concerning observed complications, mortality, and quality of life up to 5 years after the initial procedure. Furthermore, the registry will enable a compilation of evidence-based indication criteria and, in addition, also a comparison of all approved operative procedures, such as Ross or David procedures, and the use of different mechanical or biological aortic valve prostheses.

\section{Keywords}

- aortic valve disease

- registry

- aortic valve replacement

- transcatheter aortic valve implantation

- database

- outcome

- quality of life

Results Since the launch of data acquisition in July 2010, almost all institutions performing aortic valve procedures in Germany joined the registry. By now, 91 sites which perform TAVI in Germany participate and more than 15,000 datasets are already in the registry.

Conclusion The implementation of new or innovative medical therapies needs supervision under the conditions of a well-structured scientific project. Up to now relevant data for implementation of TAVI and long-term results are missing. In contrast to randomized controlled trials, GARY is a prospective, controlled, 5-year observational multicenter registry, and a real world investigation with only one exclusion criterion, the absence of patients' written consent.
\end{abstract}

\section{Introduction}

The increasing prevalence of severe aortic valve diseases correlates with the increase of life expectancy in the western industrial nations. ${ }^{1,2}$ For decades, patients with symptomatic aortic valve disease are treated by aortic valve replacement (AVR) or reconstruction as the worldwide accepted "gold standard." 2 These heart operations are performed with high success and low complication rates, with respect to short-, mid-, and long-term outcomes. ${ }^{1,2}$ In Germany, nearly 12,000 patients per annum undergo isolated aortic valve surgery, performed under the use of extracorporeal circulation (ECC). ${ }^{3,4}$

For 10 years, a new and innovative technology enables aortic valve prosthesis implantation through catheter devices, first applied successfully in human by Cribier et al in 2002. ${ }^{5}$ This development raised the expectation that there might be a reorientation for the treatment of patients suffering from symptomatic aortic valve disease in the upcoming years. ${ }^{6}$ In 2009, the Deutsche Gesellschaft für Thorax-, Herz- und Gefäßchirurgie (DGTHG) (www.dgthg.de) and the Deutsche Gesellschaft für Kardiologie - Herz- und Kreislaufforschung (DGK) (www.dgk.org) published a position statement ${ }^{7}$ with the consented recommendation that transcatheter aortic valve implantation (TAVI) should only be used in selected patients with severe comorbidities, high risk for mortality, and/or contraindications for heart surgery. ${ }^{8,9}$

Catheter-based implantations can be performed through vascular (e.g., femoral or subclavian) route (v-TAVI) as well as by transapical access (a-TAVI). ${ }^{10-12}$ These novel therapeutic options differ basically from AVR in several aspects:

- Absence of median sternotomy.

- Absence of ECC. 
- Prosthesis implantation instead of AVR.

- Absence of suture lines for valve prosthesis fixation.

- Prosthesis crimping before implantation.

- Prosthesis positioning under X-ray examination.

Due to limited experience in this field, including potential influence on patients' care, there are still various unresolved questions. ${ }^{12-14}$ In particular, benefits and acute risk of implantation compared with the surgical valve replacement must be assessed under real world conditions. In addition, long-term results and quality of life $(\mathrm{QoL})$ data are lacking. ${ }^{15}$ Furthermore, long-term durability of the new prostheses are unknown, yet. ${ }^{12}$ Accordingly, to characterize these innovative devices as therapeutic options, data from a real world registry can support recommendations for reasonable indications. ${ }^{12}$ Currently, because of only few regulations for the implementation of new technologies in German hospital care, the use of TAVI in Germany has increased rapidly in the past few years. Therefore, the German registry can provide data from a large quantity of patients.

The DGTHG and the DGK initiated this registry with the attempt to sample all aortic valve procedures nationwide. The only exclusion criterion is refusal for participation by the patient.

In this article, we describe the background, the concept, and the perspective of the German Aortic Valve Registry (GARY) as a real world investigation. ${ }^{16}$ Additional information about the registry are available on its Web site: www. aortenklappenregister.de (-Fig. 1).

\section{Materials and Methods}

GARY is a prospective, controlled, multicenter registry study which enables data acquisition across a large patient population. The main investigation objects include the following:

- Variability in structural requirements, processes, and results for different aortic valve therapies.
- Identification of indication criteria for various therapeutic options (e.g., by means of risk adjustment/scoring systems).

- Detailed information on quality and safety of medical devices and performed procedures.

- Evaluation of nationwide quality of care and on the institutional level to ensure and increase quality of medical treatment.

- Changes in QoL.

- Health economic evaluation of the applied therapies.

GARY is an evaluation and comparison of an innovative medical treatment to all well-established aortic valve therapies under aspects of benefits and risks for the patient in short-, mid-, and long-term courses. Furthermore, it is hoped that the registry will lead to the development of criteria in which patient which therapeutic option is reasonable. Finally, the registry will additionally compare various procedures such as Ross and David operations or balloon valvuloplasty (BAV) and distinctions of mechanical or biological valve prostheses.

\section{Observation Time}

The study period started on July 5, 2010 and will be finished by the end of 2016. At present, the registry is scheduled to include patients up to December 2015. The protocol requests a followup each at 30 days, 1,3 , and 5 years after the initial aortic valve procedure. In this study, 3-year follow-up is scheduled for patients from 2011 to 2013 while 5-year follow-up is intended for all patients from the year 2011. Apart from the 30-day status, the other follow-up surveys are achieved by the Institute for Quality and Patient Safety (www.bqs-institut.de) as an independent institution. Due to legal data protection regulations, the follow-up is only conducted if the patient agreed by a written consent so the participating institution is authorized to export data to the registry center. Based on earlier experiences, this approach is the most feasible and, in particular, safe to obtain longitudinal data.

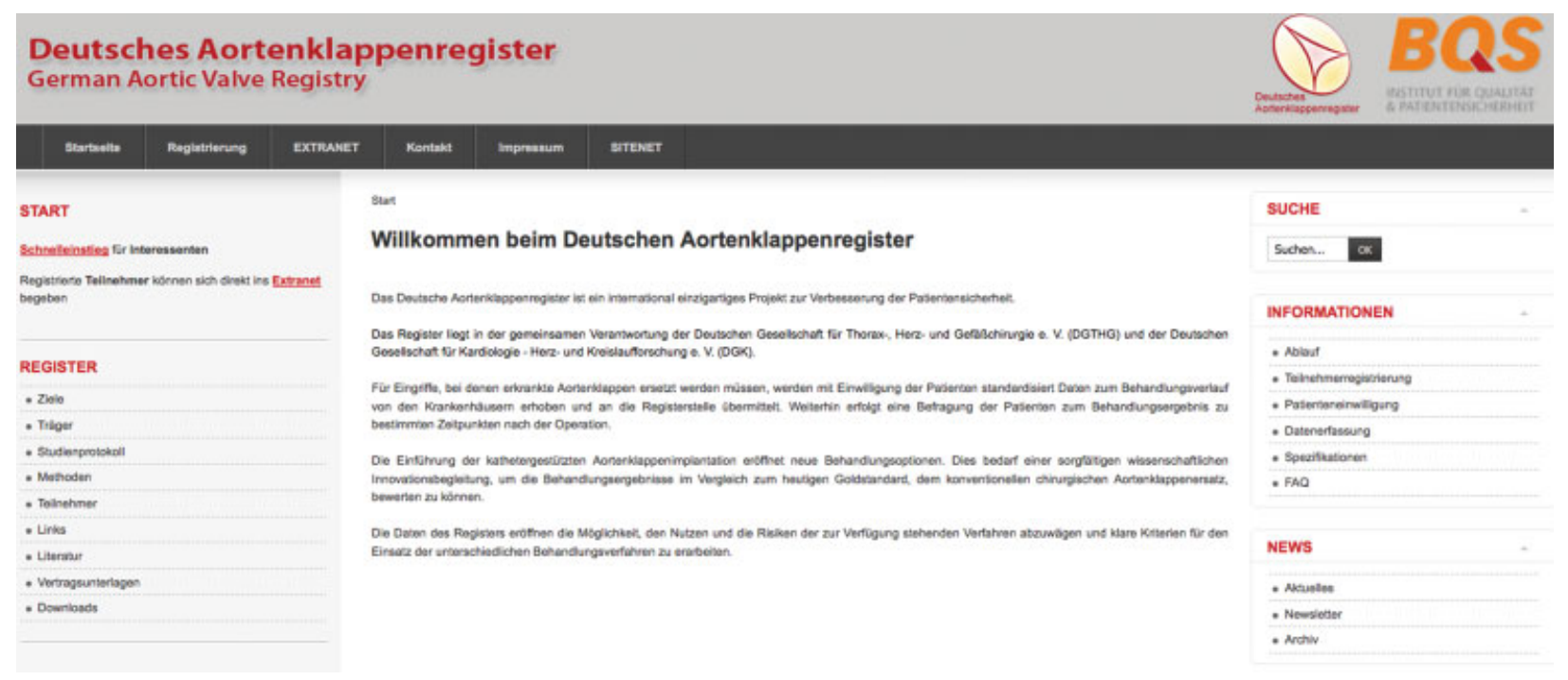

Figure 1 German Aortic Valve Registry: Public Website. 


\section{Registry Design}

All patients undergoing an invasive therapy for acquired aortic valve disease as principal indication will be consecutively included in the registry. In detail, the patient population consists of all consecutive patients with symptomatic aortic valve disease either receiving surgical AVR, aortic valve operations such as the Ross and David procedures, v-TAVI (retrograde), a-TAVI (antegrade), or aortic valvuloplasty. In case of concurrent heart diseases requiring combined procedures (e.g., coronary artery bypass grafting, mitral, or tricuspid valve surgery), the aortic valve disease by itself must fulfill the indication criteria for one of the included procedures. The only exclusion criterion is a missing compliance missing agreement for participation of the patient.

\section{Database}

GARY provides a complete survey and is based on a data model which consists of three data records from various sources (-Fig. 2). Data from the three sources are merged using patient-identifying items.

Source I is the data record of the German external quality assurance program, in accordance to the German Social Code ( 137 SGB V), in which data on pre-, intra-, and postoperative facts during hospital stay have to be collected. This source is mandatory by law for all German hospitals which perform isolated AVRs and TAVI. In fact, source I is used both for statutory requirements and for GARY because all participating centers just have to conduct an additional data transfer to the registry.

Source II is a specifically developed registry dataset which contains more specific information on the procedures, the perioperative patient status (e.g., indication criteria), and intra- and postoperative events. This dataset is obtainable as an internet-based survey or can be integrated into the medical record by a specific software tool. For each participating institution, an individual access for the online application is available. Data transfer is just possible by using a secure socket layer internet connection, and storage is performed on a server at the BQS Institute.

Source III is the follow-up survey including EQ-5D ${ }^{17}$ as a standardized questionnaire for life quality. Furthermore, a specific and predefined dataset on resource consumption is documented for further health economic analyses.

Data completeness will be verified by an electronic tool which analyses reimbursement data while data validity is monitored by a multistage plausibility check combined with an on-site data verification on a randomly selected $3 \%$ of the samples (audit).

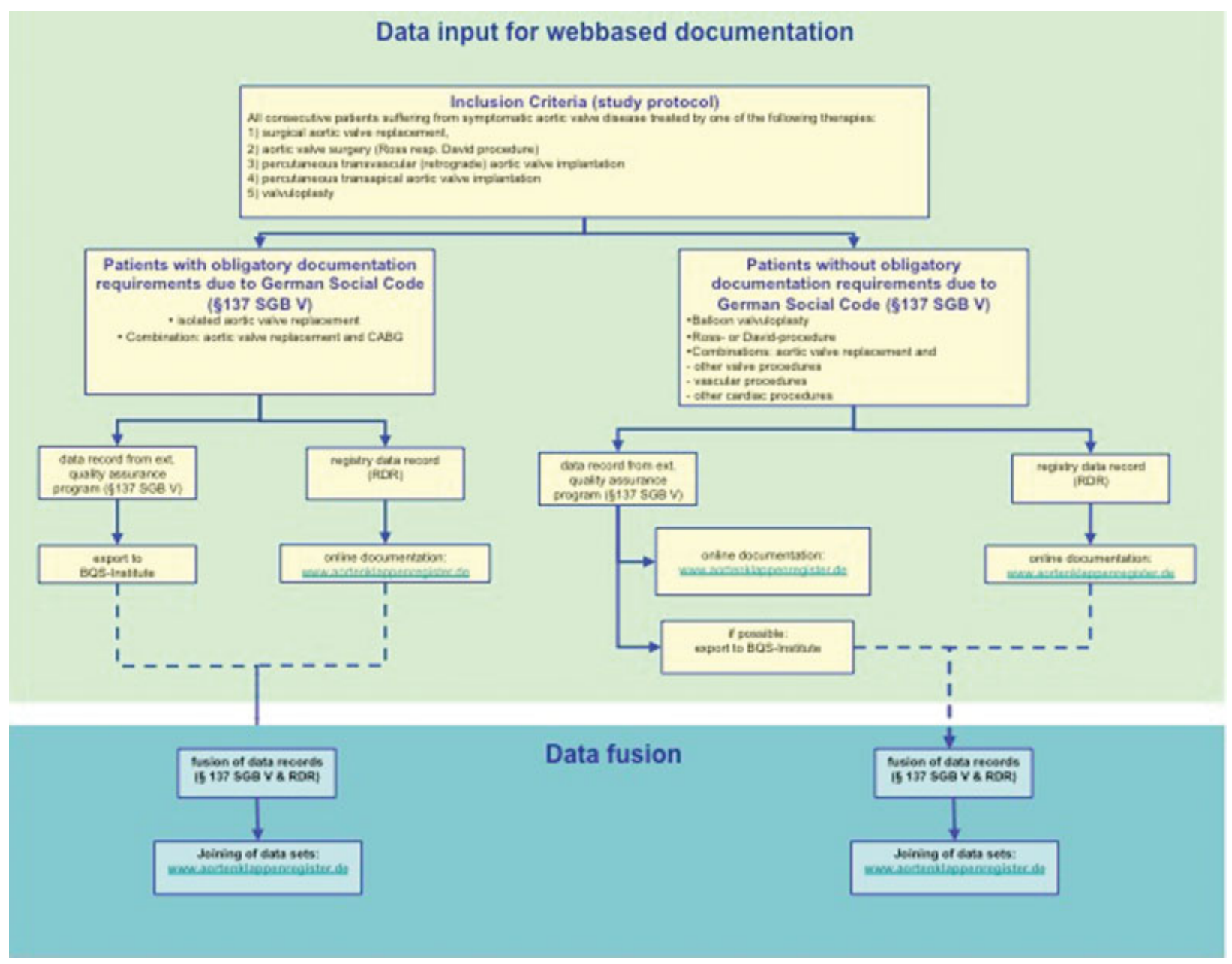

Figure 2 Data model considering different data sources. 


\section{Electronic Case Report Forms (eCRF)}

Data from all sources are assembled with eCRF. The data sheet from source I was developed by an expert group (clinical and methodical experts) under the participation of the DGTHG.

The eCRF for sources II and III were developed by the BQS Institute for Quality and Patient safety (www.bqs-institut.de) in cooperation with the members of the Executive Board (EB). All electronic data collection sheets are mostly self-explanatory. To facilitate data entry, a precise definition for appointed parameters requiring documentation can be obtained easily.

The main variables of measurement include the following:

- Event-free survival at 30 days, 1, 3, and 5 years after initial treatment.

- Eligibility criteria for obtainable procedures.

- Risk adjustment and QoL.

- Technical performance and success of intervention incl. reintervention.

- Nonfatal severe complications and in-hospital mortality.

- Medication at hospital discharge and during long-term observation.

- Applied prostheses/medical devices.

- Length of hospital stay, rehabilitation, and outpatient visits.

- Nonfatal severe complications, reintervention, rehospitalization, and mortality within 30 days, 1, 3, and 5 years.

Therefore, the following information is acquired for all patients:

- Administrative data such as registry ID, date of admission and discharge, date of procedure, and presence of consent are collected for all patients.

- QoL before and after intervention (EQ-5D).

- Hospitalizations and outpatient visits within the last 12 months.

- Preoperative findings with respect to valve disease, risk factors, and comorbidities.

- Medication at admission and discharge.

- Scoring systems: ASA-Physical Status (American Society of Anesthesiologists) NYHA-classification (New York Heart Association) CCS-classification (Canadian Cardiovascular Society).

- Duration of ventilation and duration of intensive care treatment.

- Outcome parameters.

Concerning various patients' subgroups, the registry dataset from source II has a modular design with specific parts for different therapeutic options: (1) isolated surgical AVR, (2) transcatheter interventions, and (3) BAV.

1. CRF for AVR includes the following specific parameters:

- manufacturer and type of replacement,

- mechanical support of circulation.

2. CRF for transcatheter interventions includes the following specific parameters:

- type of intervention and basis for decision,

- manufacturer and prosthesis model,
- support of circulation,

- time from incision to closure,

- fluoroscopy time,

- amount of contrast medium.

3. CRF for BAV includes the following specific parameters:

- induction of cardiac arrest by rapid pacing,

- size and type of balloon,

- subsequent ballooning and irradiation.

CRF Follow-up: This part of assessment is conducted by a phone interview performed by the BQS Institute. The dataset comprises the following information:

- Events: death, aortic valve reintervention, coronary bypass surgery, myocardial infarction, stroke, thromboembolic events, severe bleedings (requiring transfusion) percutaneous coronary intervention, implantable cardio-defibrillator/ pacemaker, dialysis, wound dehiscence.

- Symptomatic: CCS, NYHA, and occurrence of syncope.

- Adjuvant therapy at the point of follow-up.

- Questions regarding QoL (EQ-5D).

- Echocardiography (only with clinical follow-up).

- Number of hospitalizations.

- Rehabilitation measures.

- Dependence on long-term care (care level).

- Employment as well as disability and reduction of earning capacity.

It is self-evident that the information of the central phone interviews is placed at the disposal to the participants.

\section{Statistics}

For the entire study population, all collected parameters undergo a descriptive analysis for each of the therapeutic options. Survival and event-free survival will be visualized by Kaplan-Meier graphs. The descriptive analysis of main investigation objects helps to illustrate the quality of the various therapies focusing on structure, process, and outcome.

It is intended to develop a risk-adjustment model under current methodical considerations to enable a comparison of all participating institutions. ${ }^{18,19}$

Through regression models it will be possible to ascertain predictors for surgery or intervention without complications, mortality, and improvement of life quality. The results of these calculations will be used to develop criteria, that is, in which patient subgroup which techniques may achieve the best outcome.

Within the various treatments, different medical devices are used and will be compared under aspects of relation to QoL or mortality. In this context propensity-score models will be applied for adjustment purposes.

In addition to the descriptive comparisons, all complications will be analyzed under consideration of risk adjustment and reported for each participant and amended by the aspect of an institution-related effect. ${ }^{19,20}$ 
For health economic evaluation, the following data are included:

- Demographic data.

- Interventional details.

- Intraprocedural complications.

- Clinical outcome parameters.

- QoL (EQ-5D, standardized questionnaire).

- Medication.

- Quantity of consulting general practitioners and/or specialists.

- Number of hospitalizations.

- Rehabilitation measures.

- Dependence on long-term care (care level).

- Employment as well as disability and reduction of earning capacity.

A price for the consumption of resources will be determined after data collection. This enables a monetary value for consumption of resources and calculation of the incurred expenses. In this context, it might be necessary to incorporate a time factor in consumption by a country- and sector-specific inflation cost adjustment in relation to a base year. This provides the possibility of cost analysis with regard to the performed procedure. Furthermore, a cost-effectiveness analysis in which the costs of the procedures are related to the clinical effects achieved in a cost-effectiveness relation (incremental costeffectiveness ratio) will be performed. Additionally, a cost-utility analysis will be conducted wherever possible. Thereby treatment costs will be put in relation to quality adjusted life years, a combined endpoint consisting of life quality and length of life.

\section{Data Protection and Management}

German law enforces strict rules for data protection. Therefore, patients' addresses are stored separate from medical data. It is guaranteed that just authorized personnel in charge of phone interview has a strictly limited access to addresses of individual patients. Statistical analyses are only conducted with anonymized medical patient data. All personal data will be anonymized immediately after import to the database and will be deleted after finishing the last scientific analysis.

For transmission of the datasets according to German Social Code ( $§ 137$ SGB V), a specific tool is available for all participants. The tool creates a file which includes all identification characteristics such that the merging of subdatasets for each patient becomes possible. In addition, this method ensures that the registry just receives data in case of written consent of the patient.

\section{Reporting}

All participating sites will receive a yearly benchmark report in which the data and results of the individual site will be compared with the entire registry data. The report will distinguish between the various procedures for aortic valve diseases. The collected data will be analyzed under aspects of patient characteristics, performed therapy, several events, and clinical outcome. This benchmark reporting enables internal quality assurance at each site.
Annually public reports and device specific analyses will be available. In addition, specific scientific papers will be published which focus on different facts such as method and product comparisons. In addition, predictive models will be developed to identify which patient groups show most benefit by which techniques. All participants have the opportunity to submit proposals for further scientific analyses. The decision on the particular analysis is taken by the EB.

\section{Responsible Body of the Registry}

The responsible body of the registry is a nonprofit organization named Deutsches Aortenklappenregister gGmbH founded by the DGTHG and the DGK. The company is registered in Berlin. Therefore, from a legal point of view both the scientific societies are the legal data holder and owner. The general as well as the scientific management of the registry is delegated to the EB, consisting of members from both societies and various experts in an advisory capacity. All EB members have to disclose possible conflicts of interest. In addition, the organization has a steering committee in which representatives from all parties of the health care system such as medical companies, health insurance funds, national health authorities, ministerial bodies, and further relevant institutions are invited to actively participate in the registry.

\section{Funding and Independence of Registry}

The responsible societies and the BQS Institute are by virtue of their constitutions independent organizations from the legal as well as the scientific point of view. The registry receives financial support in the format of unrestricted grants by medical companies. A disclosure stipulation ensures that evaluations and publications are conducted solely according to scientific criteria. The medical companies have no access to data or their reporting.

\section{Results}

Since the launch of data acquisition on July 5, 2010, almost all sites performing aortic valve procedures joined the registry. By now nearly all institutions ( $n=91$ ) which perform TAVI in Germany participate and already more than 15,000 patients' datasets are included in the registry.

There will be annual standardized reports for all sites in which the results of each institution are compared with the data of the registry. These analyses will allow benchmarking and provide continuous quality improvement for each participating institution.

\section{Discussion}

The implementation of new and innovative medical therapies requires postmarket supervision under conditions of a wellstructured scientific project. The GARY is a real world study and aims to get detailed and consolidated findings concerning various therapeutic options for patients with aortic valve stenosis. In comparison to other industrialized countries, the TAVI procedures increase extraordinarily rapid in Germany. In other European countries, as well as in the United States, 
the application of the new technology is very cautious and restricted to few centers and narrow patient populations. Recommendations from leading medical societies of various industrialized nations agreed that the application of TAVI is still experimental and should be restricted to selected patients subsets. Especially, in the interest of patient safety data for short-, medium-, and long-term outcomes are urgently needed. In this sense, the findings of the GARY will allow to address urgent questions in the field of aortic valve diseases including long-term data on various valve prostheses and for the performed procedures. In our view, there is still need for RCTs but they should be accompanied by registries such as GARY with their advantage to involve and compare larger and complete patient populations.

GARY integrates a specific and validated questionnaire for the determination of five different patterns for patients' QoL. Furthermore, standardized questions are included which address the resource consumptions. In our opinion, QoL and health economic data are essentials, which should not be missed in a well-designed registry.

The main goal of the ambitious project "GARY" is to collect and evaluate detailed information from all patients undergoing invasive treatment for acquired aortic valve diseases. There is a longstanding tradition in Germany that makes it realistic to achieve this goal. Since decades for the "heart medicine" in Germany it is self-evident that comprehensive data collections are conducted for scientific purposes. In addition, the mandatory quality assurance according to the German law provides a framework for data transmission and completeness count. The experience since the start of GARY already demonstrates that nearly all patients agree to participation.

\section{References}

1 Iung B, Baron G, Butchart EG, et al. A prospective survey of patients with valvular heart disease in Europe: The Euro Heart Survey on Valvular Heart Disease. Eur Heart J 2003;24(13):1231-1243

2 Vahanian A, Baumgartner $\mathrm{H}$, Bax J, et al; Task Force on the Management of Valvular Hearth Disease of the European Society of Cardiology; ESC Committee for Practice Guidelines. Guidelines on the management of valvular heart disease: The Task Force on the Management of Valvular Heart Disease of the European Society of Cardiology. Eur Heart J 2007;28(2):230-268

3 Funkat A, Beckmann A, Schiller W, et al. Cardiac surgery in Germany during 2011: a report on behalf of the German Society for Thoracic and Cardiovascular Surgery. J Thorac Cardiovasc Surg 2012 (In press)

4 AQUA-Qualitätsreport 2010: Aortenklappenchirurgie isoliert (konventionell/kathetergestützt); http://www.sqg.de/themen/ qualitaetsreport/qualitaetsreport-2010/index.html

5 Cribier A, Eltchaninoff H, Bash A, et al. Percutaneous transcatheter implantation of an aortic valve prosthesis for calcific aortic stenosis: first human case description. Circulation 2002;106(24): 3006-3008

6 Vassiliades TA Jr, Block PC, Cohn LH, et al; Society of Thoracic Surgeons (STS); American Association for Thoracic Surgery (AATS); Society for Cardiovascular Angiography and Interventions (SCAI); American College of Cardiology Foundation (ACCF);
American Heart Association (AHA). The clinical development of percutaneous heart valve technology: a position statement of the Society of Thoracic Surgeons (STS), the American Association for Thoracic Surgery (AATS), and the Society for Cardiovascular Angiography and Interventions (SCAI). Ann Thorac Surg 2005;79 (5):1812-1818

7 Figulla HR, Cremer J, Walther T, et al. Positionspapier zur kathetergeführten Aortenklappenintervention. Kardiologe 2009;3:199206 (DOI 10.1007/s12181-009-0183-4)

8 Smith CR, Leon MB, Mack MJ, et al; PARTNER Trial Investigators. Transcatheter versus surgical aortic-valve replacement in highrisk patients. N Engl J Med 2011;364(23):2187-2198

9 Leon MB, Smith CR, Mack M, et al; PARTNER Trial Investigators. Transcatheter aortic-valve implantation for aortic stenosis in patients who cannot undergo surgery. N Engl J Med 2010; 363(17):1597-1607

10 Ferrari E, von Segesser LK. Transcatheter aortic valve implantation (TAVI): state of the art techniques and future perspectives. Swiss Med Wkly 2010;140:w13127

11 Holmes DR Jr, Mack MJ; Writing Committee. Transcatheter valve therapy: a professional society overview from the American College of Cardiology Foundation and the Society of Thoracic Surgeons. Ann Thorac Surg 2011;92(1):380-389

12 Holmes DR Jr, Mack MJ, Kaul S, et al. 2012 ACCF/AATS/SCAI/STS expert consensus document on transcatheter aortic valve replacement. J Am Coll Cardiol 2012;59(13):1200-1254

13 Vahanian A, Alfieri O, Al-Attar N, et al; European Association of Cardio-Thoracic Surgery; European Society of Cardiology; European Association of Percutaneous Cardiovascular Interventions. Transcatheter valve implantation for patients with aortic stenosis: a position statement from the European Association of CardioThoracic Surgery (EACTS) and the European Society of Cardiology (ESC), in collaboration with the European Association of Percutaneous Cardiovascular Interventions (EAPCI). Eur Heart J 2008; 29(11):1463-1470

14 Figulla L, Neumann A, Figulla HR, Kahlert P, Erbel R, Neumann T. Transcatheter aortic valve implantation: evidence on safety and efficacy compared with medical therapy. A systematic review of current literature. Clin Res Cardiol 2011;100(4): 265-276 Review

15 Makkar RR, Fontana GP, Jilaihawi H, et al; PARTNER Trial Investigators. Transcatheter aortic-valve replacement for inoperable severe aortic stenosis. N Engl J Med 2012;366(18):1696-1704

16 Walther T, Blumenstein J, Mohr FW, Hamm C. Aortenklappenregister: Hintergründe, Akteure und Ziele Z Herz- Thorax- Gefäßchir 2011;25:87-91

17 Szende A, Oppe M, Devlin N, eds. EQ-5D Value Sets: Inventory, Comparative Review and User Guide. EuroQol Group Monographs, Vol 2. Berlin, Heidelberg: Springer; 2007

18 Rosenhek R, Iung B, Tornos P, et al. ESC Working Group on Valvular Heart Disease Position Paper: assessing the risk of interventions in patients with valvular heart disease. Eur Heart J 2012;33(7): $822-828,828 \mathrm{a}, 828 \mathrm{~b}$

19 Leon MB, Piazza N, Nikolsky E, et al. Standardized endpoint definitions for Transcatheter Aortic Valve Implantation clinical trials: a consensus report from the Valve Academic Research Consortium. J Am Coll Cardiol 2011;57(3):253-269

20 Tommaso CL, Bolman RM III, Feldman T, et al; American Association for Thoracic Surgery; Society for Cardiovascular Angiography and Interventions; American College of Cardiology Foundation; Society of Thoracic Surgeons. Multisociety (AATS, ACCF, SCAI, and STS) expert consensus statement: operator and institutional requirements for transcatheter valve repair and replacement, part 1: transcatheter aortic valve replacement. J Thorac Cardiovasc Surg 2012;143(6):1254-1263 